\title{
openheart Heart rate variability analysis during head-up tilt test predicts nitroglycerine- induced syncope
}

\author{
Kristian Efremov, Donatella Brisinda, Angela Venuti, Emilia lantorno, \\ Claudia Cataldi, Francesco Fioravanti, Riccardo Fenici
}

To cite: Efremov K, Brisinda D, Venuti A, et al. Heart rate variability analysis during head-up tilt test predicts nitroglycerineinduced syncope. Open Heart 2014;: : 000063 .

doi:10.1136/openhrt-2014000063

Received 18 February 2014 Revised 6 May 2014 Accepted 28 May 2014

\section{CrossMark}

Catholic University of the Sacred Heart, Biomagnetism Center, Clinical Physiology, Rome, Italy

\section{Correspondence to} Professor Riccardo Fenici; feniciri@rm.unicatt.it

\section{ABSTRACT}

Objective: The aim of this study was to determine whether or not heart rate variability (HRV) analysis during the first 20 min of head-up tilt testing could predict whether patients will develop syncope after nitroglycerine administration.

Design: 64 patients with previous loss of consciousness underwent head-up tilt testing with the Italian protocol, which involves the administration of nitroglycerine after 20 min of tilt. HRV parameters were analysed from 5 min intervals selected during pretest supine rest (phase 1), the first 5 min (phase 2) and the last 5 min (phase 3 ) of passive 20 min of tilting, prior to the administration of nitroglycerine. Differences in power $\left(\mathrm{ms}^{2}\right)$ of the spectral components between the various phases of tilting were calculated for each patient and expressed as $\Delta$.

Results: 20 patients (group 1, 9 women, mean age $43.2 \pm 24.5$ years) had a syncope during tilt testing after nitroglycerine, while the other 44 (group 2, 24 women, mean age $41 \pm 20.5$ years) did not. In group 1 , the HRV spectral parameters high frequency (HF) and total power (TP) had a significant decrement from phases 2 to $3(p=0.012$ and 0.027 , respectively), while in group 2 the average HF and TP values did not change. The $\Delta$ of spectral parameters between phases 2 and 3 were able to differentiate between the two groups and to predict syncope after nitroglycerine administration $(p<0.05)$.

Conclusions: HRV analysis within the first 20 min of passive tilting demonstrated that patients with nitroglycerine-induced syncope are characterised by a progressive decrement of parasympathetic activity, which does not occur in patients with a negative response to nitroglycerine. If confirmed on a wider population, HRV analysis could replace nitroglycerine administration and shorten the duration of the tilt test.

\section{OBJECTIVE}

Head-up tilt testing (HUT) has a central part in the diagnosis of vasovagal syncope (VVS). ${ }^{1}$

Different protocols have been established over the years, with different strengths and weaknesses. $^{2-4}$ The Italian protocol has the highest sensitivity, ${ }^{5}{ }^{6}$ but has at times been

\section{KEY MESSAGES}

- Heart rate variability parameters during the first 20 minutes of head up tilt testing respond differently in head-up tilt testing (HUT) positive and negative patients.

- Heart rate variability analysis might be useful to decrease the duration and eliminate the use of nitroglycerin during head up tilt testing.

- Heart rate variability analysis can predict the outcome of a head up tilt test.

criticised for lower specificity due to the administration of nitroglycerine. ${ }^{5} \quad 7 \quad 8$ Moreover, in a very recent study carried out in patients with likely VVS, HUT with nitroglycerine had a low-diagnostic value in predicting long-term outcomes, suggesting that HUT is of limited value in the management of patients with suspected neurally mediated syncope. $^{8}$

Through the analysis of the heart rate variability (HRV), a physiological phenomenon resultant from the dynamic modulation of HR by the interaction of the sympathetic and parasympathetic nervous systems activity, it is possible to study the dynamics of event-induced changes of cardiac autonomic modulation. ${ }^{9}{ }^{10}$ Thus, HRV analysis is a powerful, non-invasive tool to explore dynamic interactions between physiological, mental, emotional and behavioural processes, whose interplay can also be pivotal in the genesis of syncopal attacks and has been used to evaluate autonomic adaptation/reaction induced by HUT. ${ }^{11-15}$

Previous studies have shown contradictory HRV results during HUT in patients with VVS and healthy controls. Most authors agree that HUT induces a decrease in high frequency $(\mathrm{HF})$ power, calculated in absolute $\left(\mathrm{ms}^{2}\right)$ and/or normalised units $(\mathrm{nu})$, variable changes of the low frequency (LF) component and an increase in the LF/HF ratio both in patients with VVS and controls. ${ }^{11} 13$ 
Other authors, however, have reported inadequate sympathovagal balance in response to orthostatism with a decrease in LF/HF in patients with positive HUT. ${ }^{16}$

In two studies, evidence has been provided that the outcome of HUT can be predicted before the onset of syncope through simultaneous HRV and blood pressure measurements, which could reduce the duration of the tilt testing and avoid induction of unnecessary full syncopal events, while maintaining the diagnostic accuracy. ${ }^{7} 17$

The aim of this study was to evaluate if HRV analysis performed before the administration of nitroglycerine could differentiate between negative and positive responders to HUT, carried out according to the Italian protocol.

\section{DESIGN}

\section{Subjects}

Sixty-four patients (31 men, 33 women, mean age 41.7 (SD 21.7) years) who had experienced at least one episode of spontaneous loss of consciousness during the previous year, most likely caused by VVS, were enrolled in the study. All of them underwent medical examinations based on the ESC guidelines. Patients with diabetes and movement disorders such as Parkinson's disease and multiple systemic atrophy, ischaemic cardiomyopathy and previous myocardial infarction were excluded, because the HRV pattern could have been affected by their underlying disease. Drugs which interact with autonomic modulation were discontinued for at least five half-lives before the day of HUT.

All patients gave their written consent to participate before enrolment in the study.

\section{ECG recordings}

All recordings were performed with a 12-lead ECG telemetric system (Mortara-Rangoni Instruments). Owing to certain factors, such as circadian rhythm, body position, activity level prior to recording, medication, verbalisation and breathing condition, which may influence the HRV, special precautions were taken to maintain similar conditions in all patients, such as performing the HUT session at a similar time of the day (usually in mid-late morning) and having the resting basal recordings after an adaptation time of at least $20 \mathrm{~min}$.

\section{Head-up tilt test}

HUT was performed according to the Italian protocol in a quiet room at a stable temperature of about $24^{\circ} \mathrm{C}$. The tilt table was electrically driven and equipped with a footplate support. After a resting period of $20 \mathrm{~min}$ in the supine position under continuous ECG monitoring, the patient was tilted to $60^{\circ}$ for $20 \mathrm{~min}$ (the so-called passive phase). Thereafter, in the absence of spontaneous syncope, $300 \mu \mathrm{g}$ of nitroglycerine spray were administered sublingually, and the patient remained tilted for an additional $20 \mathrm{~min}$ (provocation phase), unless syncope occurred.
The test was considered completed either when the patient experienced syncope, which required that the patient be immediately brought in the supine position, or at the end of the provocation phase in the absence of symptoms. The haemodynamic response to HUT was classified according to the VASIS classification.

On the basis of the results of HUT, the patients were divided into two groups. Group 1 consisted of 20 patients (11 men, 9 women, mean age 43.2 (24.5 SD)) who experienced syncope during HUT, while group 2 involved 44 patients (20 men, 24 women, mean age 41 (20.5 SD)) with a negative response to HUT.

\section{Heart rate variability}

Quantitative HRV analysis was performed as follows according to the guidelines of the European Society of Cardiology and the North American Society of Pacing and Electrophysiology. ${ }^{9}$ The first raw ECG data were extracted from the telemetric recording with a custom software routine and edited to manually remove technical and/or physiological artefacts. The fraction of total RR intervals labelled as normal-to-normal (NN) intervals was used as a measure of data reliability, with the purpose of excluding records with a ratio less than a $90 \%$ threshold. Then further editing was performed by visual analysis of the tachogram and of the corresponding ECG, with manual correction of the possible residual artefacts. Finally, HRV parameters were calculated in the time domain (TD), frequency domain (FD) and with non-linear methods using the Kubios HRV software (V.2.1). ${ }^{18}{ }^{19}$ Arbitrary criteria chosen to optimise the selection of the time-windows used for calculation were: (1) the highest possible 'stationarity' of the RR signal (defined as the absence of arrhythmia and of any kind of artefacts in a visual analysis of the corresponding ECG recordings) and (2) the best coherence among spectral output obtained with the Fast Fourier Transform (FFT) and autoregressive (AR) models. LF and HF in nu and the $\mathrm{LF} / \mathrm{HF}$ ratio were calculated from the absolute values of LF and HF. These values were used as indexes of sympathovagal interaction to explore autonomic modulation. ${ }^{2021}$

All measurements were performed from three shortterm (5 min) time intervals selected: (1) during the basal rest phase in the supine position, just before the start of tilting (phase 1), (2) during the first $5 \mathrm{~min}$ of HUT (phase 2) and (3) during the last 5 min before the administration of nitroglycerine (phase 3; figure 1).

In order to evaluate if patients who experienced syncope and those who did not had a different autonomic modulation, the differences in power $\left(\right.$ in $\mathrm{ms}^{2}$ ) of the spectral components between the various phases of the tlt were calculated for each patient and expressed as $\Delta$ (ie, $\Delta$ LF2-LF1, $\Delta$ LF3-LF2, etc). Prior to quantitative analysis, the VLF component was removed using the 'smoothing prior' method provided by the Kubios software, with the $\lambda$ value set to 400 . Measurement of LF 


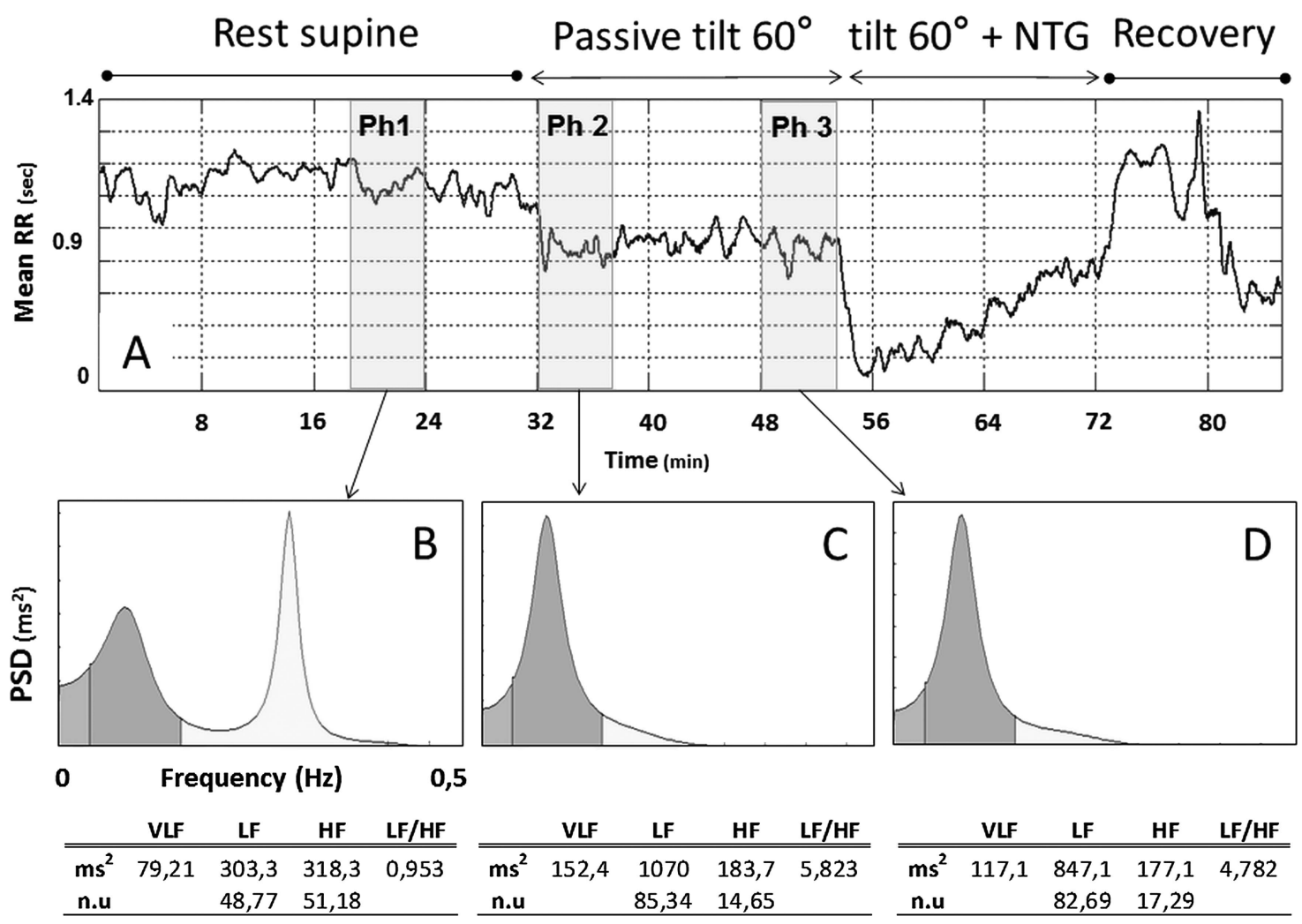

Figure 1 Example of sequential spectral analysis during the tilt test. Boxes on the tachogram (A) indicate the three 5 min epochs (Ph1: basal rest supine; Ph2: onset of passive tilt and Ph3: end of passive tilt) when HRV parameters are calculated. Spectral analysis evidence of physiological prevalence of the vagal (HF) component in the supine position (B), and its decrease (with concomitant increase of the LF component) during the subsequent phases ( $C$ and $D)$ of the study (HF, high frequency; HRV, heart rate variability; LF, low frequency; nu, normalised units). NTG, nitroglycerin; PSD, power spectral density; RR, interval between subsequent $R$ waves.

and HF power components was made in absolute values of power $\left(\mathrm{ms}^{2}\right)$, and in nu.

\section{Statistical analysis}

Statistical analysis was performed using Sigmastat 3.5 (Systat) or SPSS V.13.0 (SPSS Inc, Chicago, Illinois, USA) software. In order to evaluate if there were differences in the HRV values of each individual group between the three phases of HUT, the paired t test or the Wilcoxon Rank Sum test was used, based on the normality of data distribution.

The $\Delta$ of HRV parameters between the different groups were compared, as appropriate, with the twosided unpaired t test or the Mann-Whitney Rank Sum Test. A two-tailed $\mathrm{p}$ value $<0.05$ was considered statistically significant. Continuous data are expressed as mean \pm 1 SD.

Linear Discriminant Analysis (LDA) was used to evaluate if HRV parameters were adequate to provide a separation between group 1 and group 2 patients. LDA searches for linear combinations of the input features that can provide an adequate separation between the two patient groups. The discriminant functions used by LDA are built up as a linear combination of the variables that seek to maximise the differences between the groups. ${ }^{22}$ The classification accuracy of the method is defined as the ability to discriminate between the two investigated groups.

\section{RESULTS}

There were no significant differences between the positive (group 1) and negative (group 2) responders to HUT in terms of age and sex. Haemodynamic parameters at rest were also similar between the two groups (table 1). No significant differences in the mean RR

Table 1 Demographics and physiological data

\begin{tabular}{llll}
\hline & Group 1 & Group 2 & p Value \\
\hline Group size & 20 & 44 & \\
Age & $43.2 \pm 24.5$ & $41 \pm 20.5$ & NS \\
Male & $11(55 \%)$ & $20(45 \%)$ & NS \\
Female & $9(45 \%)$ & $24(55 \%)$ & NS \\
HR $(b p m)$ & $63 \pm 8$ & $66 \pm 10$ & NS \\
SBP (mm Hg) & $122 \pm 17$ & $117 \pm 17$ & NS \\
DBP (mm Hg) & $75 \pm 9$ & $71 \pm 9$ & NS \\
\hline DBP, diastolic blood pressure; NS, not significant; SBP, systemic \\
blood pressure.
\end{tabular}


Figure 2 Box plot of the high frequency (HF) power, measured in absolute values $\left(\mathrm{ms}^{2}\right)$ at the three phases. The $p$ level of significance between the different phases is shown for each group of patients.

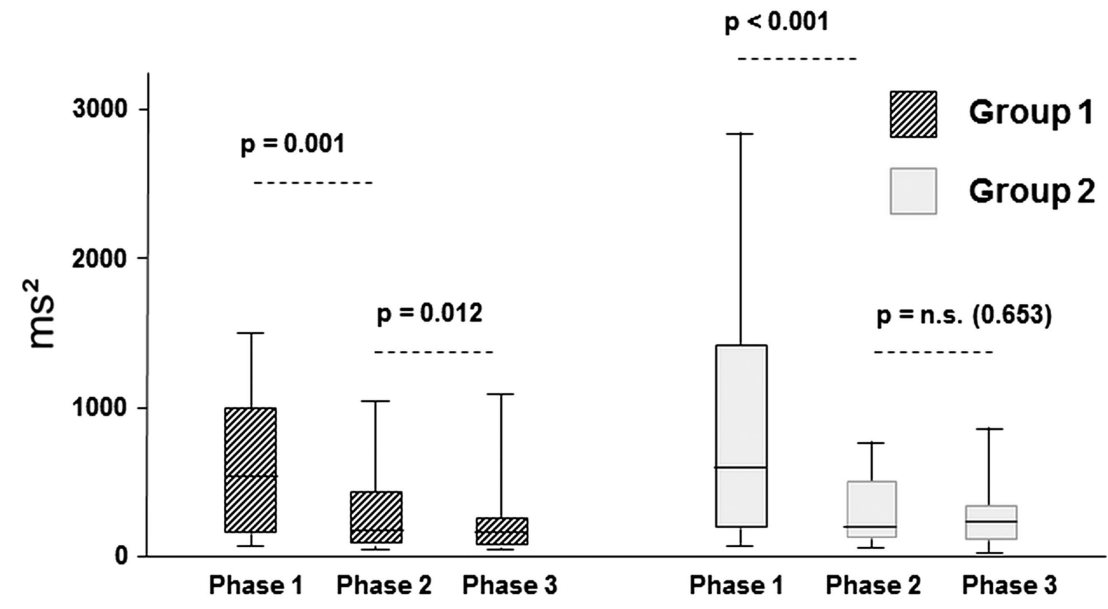

intervals in any phase, or in the $\Delta$ mean RR, were found between the two patient groups.

No patient had syncope during the first $20 \mathrm{~min}$ of passive tilt. On average, a positive response to HUT occurred at about $4 \mathrm{~min}$ and $30 \mathrm{~s}$ (median 4.5 (2:008:20) $\mathrm{min}$ ) after the administration of nitroglycerine.

According to the VASIS classification, 5 of the 20 patients who experienced syncope had mixed-type reflex (type 1), seven had a pure cardioinhibitory response (type 2) and eight had a vasodepressor response (type 3 ).

In phase 1, absolute values of LF, HF and total power (TP) were slightly higher in group 2 patients than in group 1 patients, but the difference was not statistically significant. No difference in the LF/HF ratio was observed between the two groups.

When comparing phases 1 and 2, a consistent and significant decrement of HF power $(\mathrm{p}<0.001)$ and increment of the $\mathrm{LF} / \mathrm{HF}$ ratio $(\mathrm{p}=0.002)$ were found in group 1 and group 2 patients (figures 2 and 3), with no significant difference between the two groups. In contrast, the behaviour of the LF power was highly variable, with approximately $60 \%$ of patients from both groups showing an increment and the remainder showing a decrement of the LF component ( $p=n . s . ;$ figure 4$)$.

The most relevant difference between the two groups was found by comparing the phase 2 and phase 3 parameters, where the HRV spectral components behaved differently. In fact, whereas in group 2 patients the average LF, HF and TP values did not change significantly between phases 2 and 3 , in group 1 patients the average $\mathrm{HF}$ and TP values showed a significant decrease ( $p=0.012$ and 0.027 , respectively; figures 2 and 5$)$. The average LF value of group 1 also decreased, but this change was not significant ( $p=0.078$; figure 4$)$, because in $30 \%$ of the patients the LF component increased. This variable behaviour of the LF component was apparently unrelated to the mechanism of syncope, since three of the six patients with an increase in the LF component had cardioinhibitory syncope, two had vasodepressor syncope and one had a mixed-type response. Among the patients with a decrease in the LF component, four had cardioinhibitory syncope, six had

Figure 3 Box plot of the low Group 1 frequency/high frequency (LF/HF) ratio at the three investigated phases (n.s., not significant).

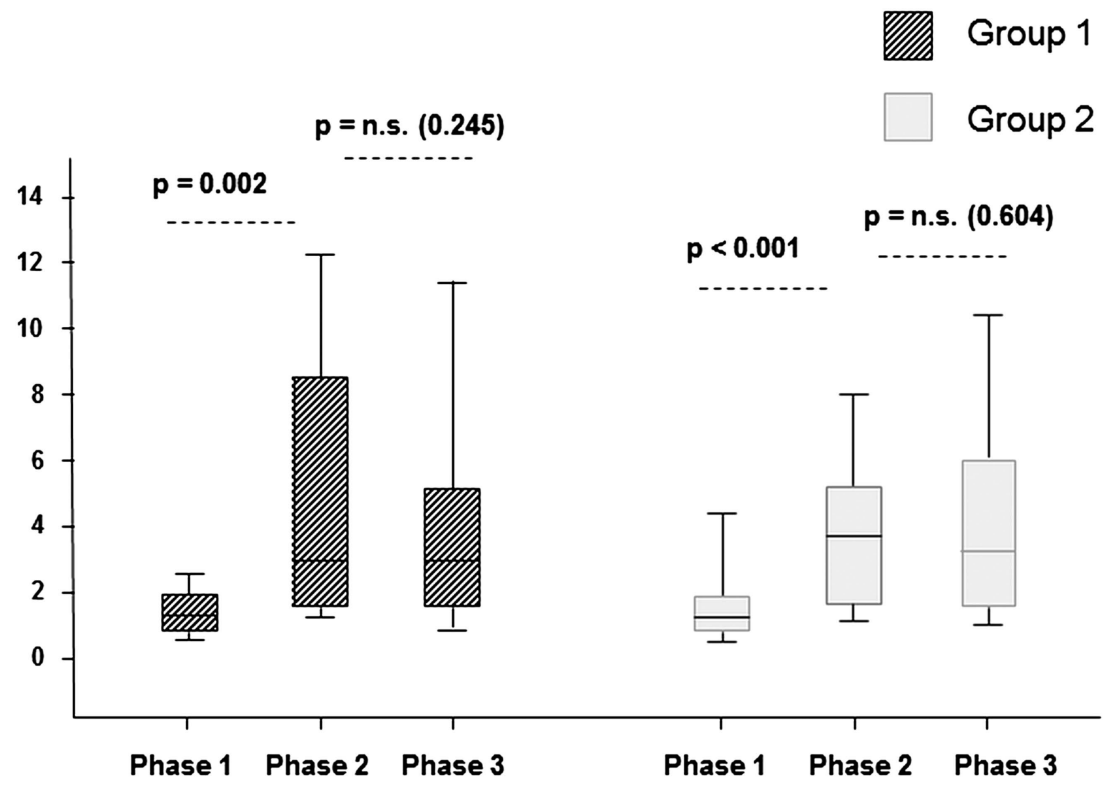


Figure 4 Box plot of the low

non-significant (n.s.), a trend towards reduction of LF power between phases 2 and 3 was observed in group 1 patients.

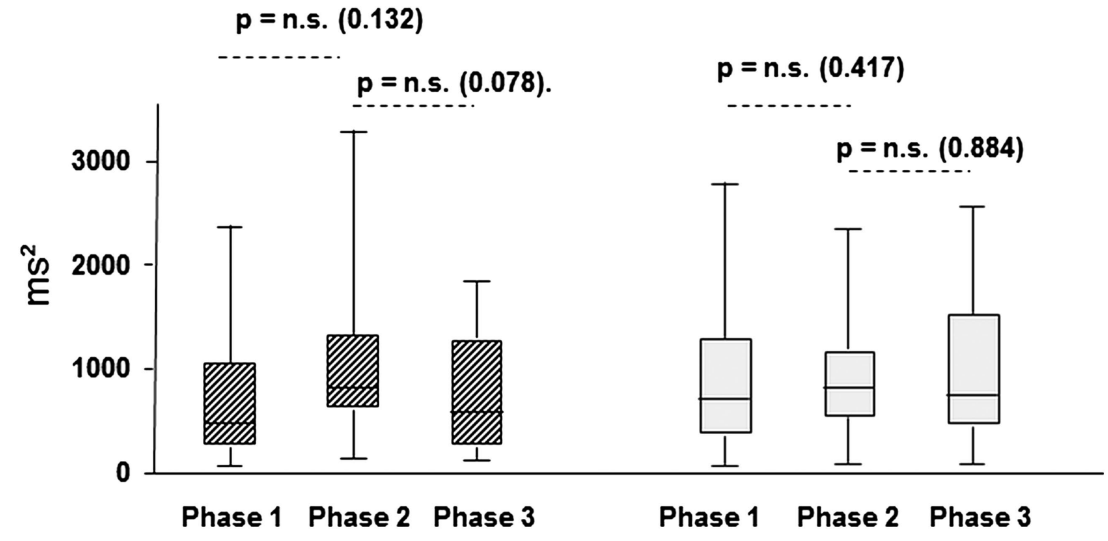

vasodepressor syncope and four had a mixed-type response. The average $\mathrm{LF} / \mathrm{HF}$ ratio also decreased in both groups, but the difference was not significant ( $p=0.245$ and 0.604 , respectively; figure 3 ).

As concerns the $\Delta$ of LF, HF and TP between the different phases, those between phases 1 and 2 were not predictive of syncope during HUT. In contrast, the $\Delta$ of spectral parameters between phases 2 and 3 were able to differentiate between the two groups and to predict syncope after nitroglycerine administration (figure 6).

Certain time-domain and non-linear HRV parameters also showed statistically significant changes in group 1 patients, which were consistent with the previously described changes in the spectral components. In particular, RMSSD, pNN50 and SD1 (all indices of vagal modulation, thus correlated to the HF spectral component) significantly decreased in group 1 . In contrast, no significant change in group 2 patients was found (table 2 and figure 7). In addition, SD2, an index of long-term variability and also linked to overall variability, decreased between phases 2 and 3 in group 1 patients, but remained unchanged in group 2 patients (table 2 and figure 7 ).

By applying LDA against the whole dataset of HRV parameters and the $\Delta$ between phases 2 and 3 , a good separation was obtained between group 1 and group 2 patients. The highest classification accuracy (80\%) obtained was based on the combination of features $\Delta \mathrm{HF}$, $\Delta \mathrm{LF}, \Delta \mathrm{SDNN} / \mathrm{RMSSD}, \Delta \mathrm{LF} / \mathrm{HF}, \Delta \mathrm{TP}$ and $\mathrm{HF}$ of phase 2 $\left(\mathrm{ms}^{2}\right)$, using the following equation:

$$
\begin{aligned}
& 0.012 \times \Delta \mathrm{HF}+0.006 \times \Delta \mathrm{LF}-1.115 \times \Delta \mathrm{SDNN} / \mathrm{RMSSD} \\
& +0.229 \times \Delta \mathrm{LF} / \mathrm{HF}-0.006 \times \Delta \mathrm{TP}+0.004 \times \mathrm{HF}-1.374<0
\end{aligned}
$$

This equation provided $85 \%$ sensitivity and $77 \%$ specificity in predicting nitroglycerine-induced syncope during HUT.

\section{DISCUSSION}

HUT is a standardised, non-invasive and fast method suggested by current guidelines for the diagnosis of reflex VVS. ${ }^{1}$ However, over the years, different protocols have been used to improve its sensitivity and specificity. ${ }^{1-5}$

Among others, the Italian protocol was developed to speed up the test and to increase its sensitivity, by introducing drug-mediated vasodilatation. ${ }^{4}$ However, some criticism has risen against this approach in that the administration of nitroglycerine could enhance the possibility of false-positive results. ${ }^{58}$
Figure 5 Box plot of the total power (TP).

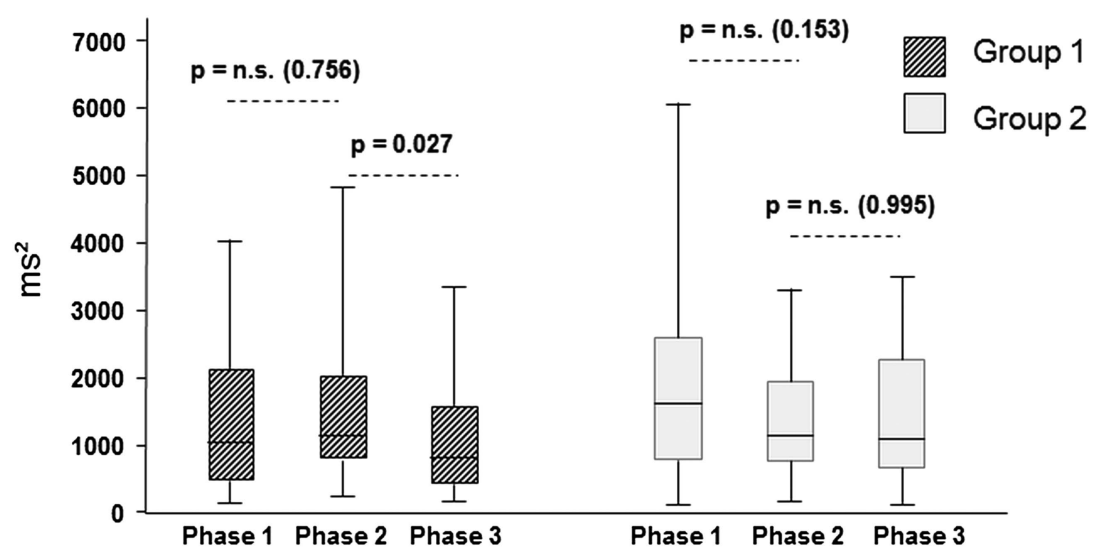




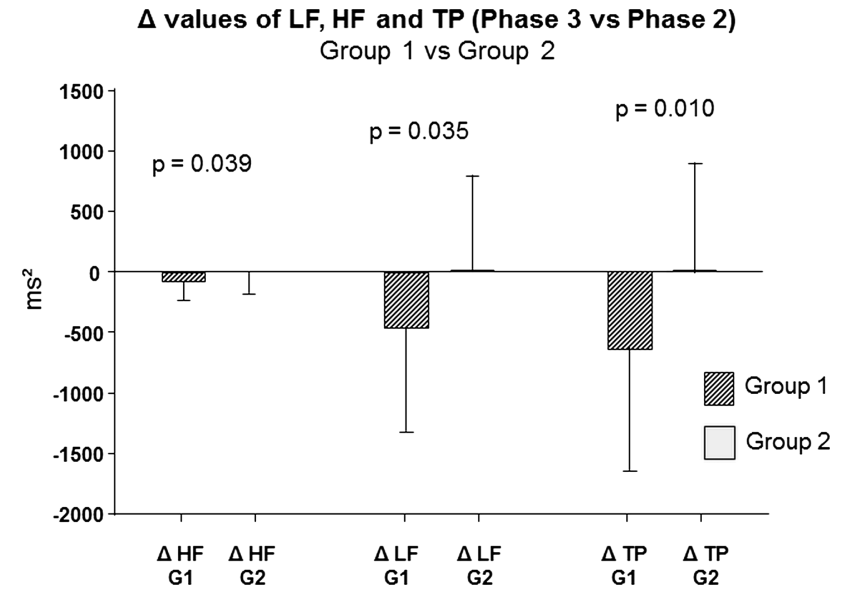

Figure 6 Different behaviours between group 1 and group 2 patients of the mean and SD of $\Delta$ for low frequency (LF), high frequency (HF) and total power (TP) calculated between the beginning and the end of the 20 min of passive tilt.

Given that HRV analysis is well recognised as a method to evaluate dynamic changes of autonomic modulation of cardiovascular function ${ }^{9}{ }^{10}$ and that the sympathetic and parasympathetic branches of the nervous system are greatly affected by the changes that occur during HUT for syncopal patients, HRV has been increasingly used in combination with HUT to better understand the mechanisms involved in the development of syncope $\mathrm{e}^{11-16}$ and to predict HUT outcome. Virag et $a l^{17}$ predicted VVS with a $95 \%$ sensitivity and 93\% specificity in 1150 patients undergoing HUT, using an algorithm based on the spectral analysis of heart rate and systolic blood pressure variability.

In the present study, we have found evidence that patients who will develop syncope after the administration of nitroglycerine according to the Italian protocol might be predicted by short-term HRV analysis carried out at the beginning and at the end of the first $20 \mathrm{~min}$ of HUT, even before the administration of the drug. In these patients, the power of HF and LF spectral components kept decreasing as the passive HUT progressed. In contrast, HRV parameters of patients who did not develop syncope with the pharmacological stimulus did not change significantly along the passive phase of HUT. Accordingly, the $\Delta$ of HF, LF and TP between the first
$\Delta$ values of RMSSD, pNN50, SD1 and SD2 (Phase 3 vs Phase 2) Group 1 vs Group 2

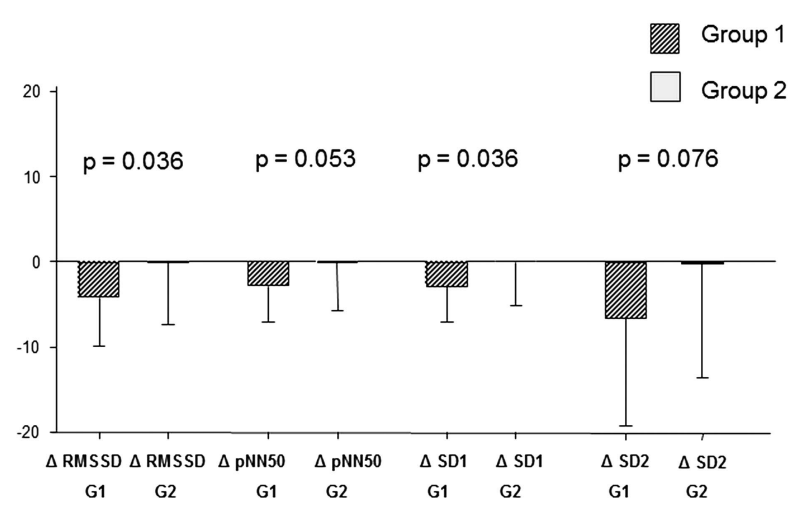

Figure 7 Statistically significant differences of the mean and SD of $\Delta$ for some time-domain and non-linear parameters between group 1 and group 2 patients.

$5 \mathrm{~min}$ of HUT and the last $5 \mathrm{~min}$ of the $20 \mathrm{~min}$ passive phase of the tilt were significantly different between group 1 and group 2 patients. These changes were consistent with changes in TD and non-linear HRV parameters, further confirming the physiological relevance of these findings.

With regard to the predictive value of HRV in differentiating between patients who will suffer a syncope at HUT, HF power has thus far shown the most consistent behaviour, with a decrease during the interval between the basal state and the beginning of the tilt observed in most studies, ${ }^{7} 132324$ a finding that was consistent with our results. Duplyakov et $a l^{7}$ and Kochiadakis et $a l^{23}$ considered the behaviour of HF from the beginning of the tilt to the period right before the end of HUT, and discovered that HF further decreases in syncopal patients, while it seems to not vary significantly in HUT-negative patients. Our results indicate that such a significant decrease in HF power can be appreciated within the first $20 \mathrm{~min}$ of HUT and that this behaviour is predictive of a positive response to nitroglycerine (ie, patients who will develop syncope), whereas in patients with a negative response to nitroglycerine-potentiated HUT the HF power remains practically unchanged.

Reports on LF behaviour from rest to tilt have been very contradictory. Some authors found that LF

Table 2 Phase-dependent changes of HRV TD and NL parameters during HUT

\begin{tabular}{|c|c|c|c|c|c|c|c|c|}
\hline & \multicolumn{2}{|l|}{ RMSSD } & \multicolumn{2}{|l|}{ PNN50 } & \multicolumn{2}{|l|}{ SD1 } & \multicolumn{2}{|l|}{ SD2 } \\
\hline & Group 1 & Group 2 & Group 1 & Group 2 & Group 1 & Group 2 & Group 1 & Group 2 \\
\hline Phase 1 & $39.5 \pm 20.6$ & $47.3 \pm 29.6$ & $11.7 \pm 15.9$ & $22.2 \pm 20.4$ & $28.0 \pm 14.6$ & $33.5 \pm 21.0$ & $44.1 \pm 22.1$ & $54.0 \pm 29.3$ \\
\hline Phase 2 & $29.1 \pm 15.7$ & $28.2 \pm 12.5$ & $9.6 \pm 11.8$ & $8.2 \pm 9.0$ & $20.6 \pm 11.1$ & $20.0 \pm 8.8$ & $47.7 \pm 21.1$ & $48.9 \pm 20.4$ \\
\hline Phase 3 & $24.9 \pm 12.5$ & $27.9 \pm 13.5$ & $6.6 \pm 9.4$ & $8.0 \pm 9.1$ & $17.7 \pm 8.8$ & $19.8 \pm 9.5$ & $41.1 \pm 19.4$ & $48.8 \pm 21.8$ \\
\hline $\mathrm{p}$ (phase 1 vs 2) & 0.003 & $<0.001$ & 0.004 & $<0.001$ & 0.003 & $<0.001$ & NS & NS \\
\hline p (phase 2 vs 3 ) & 0.004 & NS & 0.005 & NS & 0.004 & NS & 0.032 & NS \\
\hline
\end{tabular}


increases in HUT-positive and HUT-negative patients; ${ }^{7} 1213$ others reported no change in LF power in HUT-negative patients and a decrement in HUT-positive patients, ${ }^{23}$ whereas a third group observed a decrease in LF power in HUT-positive and HUT-negative patients. ${ }^{24}$ In addition, several studies reported an increase in LF in HUT-positive patients before the start of the tilt and the occurrence of syncope. $^{723}$ It has to be considered, however, that the methods used to calculate the FD metrics differ from paper to paper and that somehow different results can be obtained with the FFT and with different orders of AR models. It is therefore often difficult to compare the results and conclusions for different studies since there is often insufficient detail in the papers to determine how the power spectral density estimates are computed.

In our study, LF behaviour at the start of HUT was variable. On average, a certain decrease in LF power was observed between phases 2 and 3 in group 1 patients, which however was not statistically significant (figure 4). Accordingly, a significant $(p=0.027)$ decrease in TP was found in group 1 patients in phase 3 of HUT, which was due to the decrease in LF and HF powers (figure 5).

The LF/HF ratio usually increases on tilt for normal patients, because sympathetic stimulation manifests as an increased LF component, which entity is correlated to the degree of tilt. ${ }^{11}$ Some authors have described a higher increment in HUT-positive patients, and suggested that it can be used to predict the outcome of HUT. $^{7} 25$ Duplyakov et $a l^{7}$ managed to successfully predict the outcome of HUT using the LF/HF ratio during the first 5 min of HUT, but this occurred only in patients undergoing the Westminster protocol, not the Italian one, and using FFT for spectral analysis. ${ }^{7}$ In our study, no difference was found in the LF/HF ratio between the two groups using the AR model. ${ }^{26}$

Although changes in several individual parameters could differentiate between HUT-positive and HUT-negative patients in our study, the predictive power increased significantly when several parameters were used simultaneously. The changes in all four frequencydomain parameters, together with the $\triangle \mathrm{SDNN} / \mathrm{RMSSD}$ and the absolute value of HF during phase 2, gave us the highest predictive accuracy $(80 \%)$.

Finally, as concerns the potential use of HRV analysis to differentiate between the mechanisms of syncope, Guzman $e t a l^{27}$ suggested that a classification based on a rise or fall of individual HRV parameters was too simplistic to differentiate between patients with different types of VVS. Within their study population, it was shown that HF power increased from the basal value to the beginning of the tilt in patients who had vasodepressor syncope, that the LF/HF ratio was higher in vasodepressor syncope than in cardioinhibitory or mixed-type syncope, and that patients with cardioinhibitory or mixed VVS experience an initial rise in the $\mathrm{LF} / \mathrm{HF}$ ratio during HUT, whereas vasodepressive VVS patients experience a drop. We were not able to find any significant differences between the various types of VVS. However, this could be due not only to the well-known high degree of inter-patient variability in the LF/HF ratio, ${ }^{28}$ but also to the smaller number of patients with each type of VVS investigated in our series.

The overall number of investigated patients who experienced VVS (only 30\%) is another limitation of this study. However, we believe that this does not affect the outcome of the results, as our goal was to see the differences between patients who experience syncope during HUT according to the Italian protocol and those who do not, regardless of the origin of their loss of consciousness.

\section{CONCLUSIONS}

In this study, we found that quantitative analysis of timerelated changes of HRV parameters during the first 20 min of passive HUT can foretell the outcome of nitroglycerine-induced potentiation according to the Italian protocol without the administration of the drug, with $80 \%$ predictive accuracy. HRV analysis during the basal period and the first $5 \mathrm{~min}$ of the passive tilt was not sensitive enough to distinguish between patients who will and those who will not have syncope at HUT carried out with the Italian protocol. However, evaluation of the dynamic variation of HRV parameters during the first 20 min of passive HUT evidenced significant differences between patients who will develop syncope after nitroglycerine administration and those who will not. Further research is warranted to evaluate whether or not appropriately timed HRV analysis might improve the differentiation among different mechanisms of syncope.

Funding This research received no specific grant from any funding agency in the public, commercial or not-for-profit sectors.

Competing interests None.

Ethics approval Institutional Research Center Review Board.

Provenance and peer review Not commissioned; externally peer reviewed.

Data sharing statement No additional data are available.

Open Access This is an Open Access article distributed in accordance with the Creative Commons Attribution Non Commercial (CC BY-NC 3.0) license, which permits others to distribute, remix, adapt, build upon this work noncommercially, and license their derivative works on different terms, provided the original work is properly cited and the use is non-commercial. See: http:// creativecommons.org/licenses/by-nc/3.0/

\section{REFERENCES}

1. Moya A, Sutton R, Ammirati F, et al. Task force for the diagnosis and management of syncope (version 2009). Eur Heart $J$ 2009;30:2631-71.

2. Benditt DG, Ferguson DW, Grubb BP, et al. Tilt table testing for assessing syncope. American College of Cardiology. J Am Coll Cardiol 1996;28:263-75.

3. Fitzpatrick AP, Theodorakis G, Vardas P, et al. Methodology of head-up tilt testing in patients with unexplained syncope. J Am Coll Cardiol 1991;17:125-30.

4. Bartoletti A, Alboni P, Ammirati F, et al. 'The Italian Protocol': a simplified head-up tilt testing potentiated with oral nitroglycerin to 
assess patients with unexplained syncope. Europace 2000;2:339-42

5. Forleo C, Guida P, lacoviello M, et al. Head-up tilt testing for diagnosing vasovagal syncope: a meta-analysis. Int $J$ Cardiol 2013;168:27-35.

6. Barón-Esquivias G, Cayuela A, Pedrote A, et al. Caracteristicas clinicas y resultados del test de tabla basculante utilizando tres protocolos en 1.661 pacientes con sincope. Rev Esp Cardiol 2003;56:916-20.

7. Duplyakov D, Golovina G, Sysuenkova E, et al. Can the result of a tilt test be predicted in the first five minutes? Cardiol J 2011;18:521-6.

8. Petix NR, Del Rosso A, Furlan R, et al. Nitrate-potentiated head-up tilt testing (HUT) has a low diagnostic yield in patients with likely vasovagal syncope. Pacing Clin Electrophysiol 2014;37:164-72.

9. Task force of the European Society of Cardiology and the North American Society of Pacing and Electrophysiology. Heart rate variability: standards of measurement, physiological interpretation and clinical use. Circulation 1996;93:1043-65.

10. Gunduz $\mathrm{H}$, Talay $\mathrm{F}$, Arinc $\mathrm{H}$, et al. Heart rate variability and heart rate turbulence in patients with chronic obstructive pulmonary disease. Cardiol J 2009;16:553-9.

11. Montano N, Ruscone TG, Porta A, et al. Power spectrum analysis of heart rate variability to assess the changes in sympathovagal balance during graded orthostatic tilt. Circulation 1994;90:1826-31.

12. Lipsitz LA, Mietus J, Moody GB, et al. Spectral characteristics of heart rate variability before and during postural tilt. Relations to aging and risk of syncope. Circulation 1990;81:1803-10.

13. Mehlsen J, Kaijer MN, Mehlsen AB. Autonomic and electrocardiographic changes in cardioinhibitory syncope. Europace 2008;10:91-5.

14. Takase $B$, Bjerregaard $P$, Greenwalt $T$, et al. Heart rate variability and head-up tilt testing in patients with syncope of undetermined etiology. Jpn Circ J 1996;60:841-52.

15. Suzuki M, Hori S, Tomita $Y$, et al. Orthostatic decrease in cardiac chaos during the head-up tilt test in patients with vasovagal syncope. Circ J 2006;70:902-8.

16. Kouakam $\mathrm{C}$, Lacroix $\mathrm{D}$, Zghal $\mathrm{N}$, et al. Inadequate sympathovaga balance in response to orthostatism in patients with unexplained syncope and a positive head up tilt test. Heart 1999;82:312-18.
17. Virag N, Sutton R, Vetter R, et al. Prediction of vasovagal syncope from heart rate and blood pressure trend and variability: experience in 1,155 patients. Heart Rhythm 2007;4:1375-82.

18. Tarvainen MP, Niskanen JP, Lipponen JA, et al. Kubios HRV-heart rate variability analysis software. Comput Methods Programs Biomed 2014;113:210-20.

19. Tarvainen MP, Ranta-Aho PO, Karjalainen PA. An advanced detrending method with application to HRV analysis. IEEE Trans Biomed Eng 2002;49:172-5.

20. Lombardi F, Stein PK. Origin of heart rate variability and turbulence: an appraisal of autonomic modulation of cardiovascular function. Front Physiol 2011;2:95.

21. Montano N, Porta A, Cogliati C, et al. Heart rate variability explored in the frequency domain: a tool to investigate the link between heart and behavior. Neurosci Biobehav Rev 2009;33:71-80.

22. Melillo P, Bracale M, Pecchia L. Nonlinear heart rate variability features for real-life stress detection. Case study: students under stress due to university examination. Biomed Eng Online 2011; 7:96.

23. Kochiadakis GE, Kanoupakis EM, Igoumenidis NE, et al. Spectral analysis of heart rate variability during tilt-table testing in patients with vasovagal syncope. Int J Cardiol 1998;64:185-94.

24. Furlan R, Piazza S, Dell'Orto S, et al. Cardiac autonomic patterns preceding occasional vasovagal reactions in healthy humans. Circulation 1998;98:1756-61.

25. Ruiz GA, Madoery C, Arnaldo F, et al. Frequency-domain analysis of heart rate variability during positive and negative head-up tilt test: importance of age. Pacing Clin Electrophysiol 2000; 23:325-32.

26. Broadman A, Schlindwein FS, Rocha AP, et al. A study on the optimum order of autoregressive models for heart rate variability. Physiol Meas 2002;23:325-36.

27. Guzmán CE, Sánchez GM, Márquez MF, et al. Differences in heart rate variability between cardioinhibitory and vasodepressor responses to head-up tilt table testing. Arch Med Res 1999;30:203-11.

28. Clifford GD. Signal Processing Methods for Heart Rate Variability [thesis]. Oxford: University of Oxford, 2002:166-84. 